\title{
Erratum: Experiments of Drops Impacting a Smooth Solid Surface: A Model of the Critical Impact Speed for Drop Splashing [Phys. Rev. Lett. 113, 024507 (2014)]
}

Guillaume Riboux and José Manuel Gordillo

(Received 30 September 2014; published 30 October 2014)

Two typos appeared in the Letter. The denominator in Eq. (4) must be $2 \sigma$ instead of $\sigma$, and there is also an extra factor 2.8 in the equation for $H_{t}$ five lines below Eq. (4). These are corrected as follows:

$$
\beta=\frac{V_{v}}{V_{r}}=\left(\frac{K_{l} \mu_{g} V_{t}+K_{u} \rho_{g} V_{t}^{2} H_{t}}{2 \sigma}\right)^{1 / 2}
$$

and

$$
H_{t}=R h_{t}=2.8 R h_{a} \simeq R \sqrt{12} / \pi t_{e, \text { crit }}^{3 / 2},
$$

with $h_{a}$ deduced in the Supplemental Material. The constant 2.8 is obtained through the fitting of the experimental data in the inset of the Fig. 3(b).

We discovered these typos once Andrzej Latka pointed us out he was unable to reproduce our results. We would like to thank him, C.S. Stevens and S.R. Nagel for their interest in our work. 